\title{
Tracking Human Locomotion by Relative Positional Feet Tracking
}

\section{Conference Paper}

Author(s):

Zank, Markus; Nescher, Thomas; Kunz, Andreas (D)

Publication date:

2015

Permanent link:

https://doi.org/10.3929/ethz-a-010412926

Rights / license:

In Copyright - Non-Commercial Use Permitted

Originally published in:

https://doi.org/10.1109/VR.2015.7223423 


\title{
Tracking Human Locomotion by Relative Positional Feet Tracking
}

\author{
Markus Zank* Thomas Nescher ${ }^{\dagger} \quad$ Andreas Kunz \\ Innovation Center Virtual Reality - IWF - ETH Zurich
}

\begin{abstract}
Tracking human movements and locomotion accurately in real time - e.g. for providing real walking in virtual environments - requires expensive tracking systems which need a lot of time to install. Furthermore, the costs typically increase with the size of the tracked space as these systems rely on external references. This poster presents an approach to significantly reduce costs for tracking human locomotion in large, potentially unlimited tracking spaces.

The proposed approach employs a low-cost user-worn position and orientation tracking system to track the position and orientation of limbs including the user's feet. To associate the relative limbs' movement within the user's local coordinate system with the absolute movement in a given environment, the feet stance phase is used. Whenever a foot enters the stance phase, the local position is locked onto the global position because the foot is not moving. This allows tracking a person with all locally tracked objects - e.g. limbs, head, etc. - by temporarily locking the absolute position alternately to the user's feet in a step by step manner. This tracking works continuously and in real time without any external references.
\end{abstract}

Keywords: Tracking, head tracking, relative positional tracking, virtual reality, locomotion, zero velocity update.

Index Terms: I.3.7 [Computer Graphics]: Three-Dimensional Graphics and Realism-Virtual reality

\section{INTRODUCTION}

Real walking for navigation in virtual environments (VEs) provides a compelling user experience and was shown to be more presenceenhancing than other navigation metaphors [5]. Such VR systems are typically composed of a head mounted display (HMD) to visualize the VE, a tracking system, and a computer with some software to render the VE. The tracking system tracks the user's viewpoint in the real environment and, if required, hands, feet, and other limbs as well. However, to keep the user immersed in the VE, such systems have high requirements in terms of accuracy, latency, range, resolution, etc. This significantly increases the cost and also the installation effort.

The recent availability of low-cost consumer HMDs has greatly reduced the cost for building VR systems. Hence, the major challenge remains designing a low-cost tracking solution which fulfills the requirements for VR applications. Therefore, within this poster, a new tracking approach is proposed which can avoid the shortcomings of existing systems.

Most of today's tracking systems require setting up a static reference system such as visual markers (fiducials) in a dedicated area where the tracking is performed (the tracking space). If a person is to be tracked at a new place, the tracking space must first be equipped again with the static reference system and calibrated. Furthermore, these systems do not scale well with the size of the track-

\footnotetext{
*e-mail: zank@iwf.mavt.ethz.ch

†e-mail:nescher@iwf.mavt.ethz.ch

‡e-mail:kunz@iwf.mavt.ethz.ch
}

ing space. Other tracking systems rely on an existing infrastructure that provide a reference system. E.g. the global positioning system (GPS) enables outdoor tracking by relying on a world-wide reference infrastructure. Other techniques e.g. employ wireless network stations for localization. However, these tracking techniques usually do not provide a sufficient accuracy and precision as required by human-computer interaction or VR applications.

Ideally, a tracking system should allow for real-time 6-degreeof-freedom (6 DOF) tracking with high precision, low latency, and a high update rate independent of the tracking space's size. To achieve this and avoid expensive external references, relative tracking provides an alternative approach. With relative tracking, the tracking system and all references are attached to the tracked person. This way, the system is self-contained and can be deployed rapidly. As the tracking system only has to operate in a personsized volume, costs are reduced significantly because even lowcost tracking systems can provide a good precision and accuracy for such a small volume.

In order to infer the absolute movement of a person, the relative movement of body parts is used with regard to one initial starting point. Previously, this was done with inertial measurement units (IMUs) attached to a person's feet, see [1]. For every step, the measured acceleration is integrated to obtain the step length and direction which is added to the absolute position. However, inertial sensors suffer from drift, which increases quadratically over time. To reduce this drift error caused by sensor noise, IMU based tracking approaches require some static reference from time to time. Human locomotion allows for such a recalibration during the foot's stance phase. During this phase, the foot is not moving which implies that the velocity estimate of the sensor attached to the foot must be zero. However, other body parts cannot be tracked easily with IMUs only because no static reference is available or a large number of IMUs would have to be attached to the body. Sometimes magnetic sensors are employed to track at least the planar orientation of limbs or the head using earth's magnetic field. Hodgson et al. [3] developed such a VR system based on IMUs and magnetic sensors only. However, magnetic tracking based on earth's magnetic field is inaccurate and often not suitable for indoor use due to interferences.

Instead of using IMUs, a wearable electromagnetic tracker can be used for relative tracking. In [6, 2], a user-worn electromagnetic position tracker is used for localizing a walking user. The approach presented in this poster is also based on relative positional tracking but allows for real-time, continous tracking of various body parts including the user's viewpoint.

\section{SYSTEM}

\subsection{Hardware}

The hardware setup is based on the Razer Hydra ${ }^{1}$ electromagnetic tracker. This is a 6 DOF tracking system which is carried along by the user. It can determine the position and orientation of two sensors which are attached to the user's feet (on top of the feet tip), see Figure 1(b). The base station (emitter) is attached to the HMD, see Figure 1(a). This allows tracking the user's viewpoint with respect to his feet or vice versa.

\footnotetext{
${ }^{1}$ http://sixense.com/razerhydra
} 


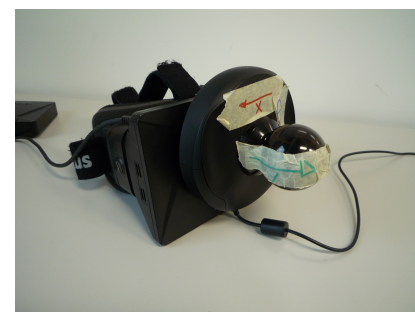

(a)

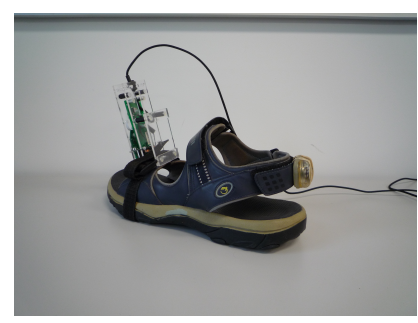

(b)
Figure 1: (a) The Razer Hydra base station attached to the HMD. (b) Foot with Razer Hydra and ReSense inertial sensor.

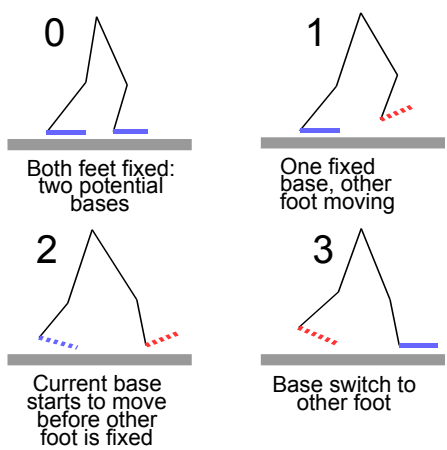

Figure 2: Step tracker phases. The current base foot is shown as a blue line, the other foot as a red line. A dashed foot line symbolizes that the foot is not fixed but moving.

The foot sensors in Figure 1(b) are modified and each has an additional Resense [4] IMU attached at the heel for detecting the stance phase similar to the setup used in [7].

\subsection{Tracking Principle}

The tracking principle is shown in Figure 2. Initially (phase 0), it can be assumed that a user is standing and both feet are fixed (stance phase). Therefore, any foot can be assigned to be the reference foot. The base allows linking the local coordinate system of the userworn tracking system with some global reference system. As soon as the user starts walking and lifts one foot (phase 1) the foot that remains fixed (in the stance phase) will be the reference. Hence, all other locally tracked points, e.g. the other foot, can also be tracked in the global reference system. Next, the reference should simply be switched when the other foot starts touching the floor. However, the stance phases of both feet do not overlap, see Figure 2. As soon as the heel of the non-reference foot starts touching the ground - but is still moving - the reference foot's heel is lifted. Hence, for a short time interval both feet are not fixed. In order to guarantee tracking during this time period, the IMU of the current reference fooot is used to estimate the base's position as described in [1]. Finally (phase 3), as soon as the non-reference foot enters the stance phase, the reference is switched and the tracking continues as in phase 1.

Using this stance phase by stance phase update, the locomotion - including any locally tracked point - of a walking person can be tracked in an absolute reference system. The tracking that provides absolute movement data runs continuously in real time and not only on a step by step basis as the base switch is done transparently.

\section{Conclusions and Future Work}

Within this poster a low-cost, self-contained tracking approach was presented which requires minimal installation or calibration effort.

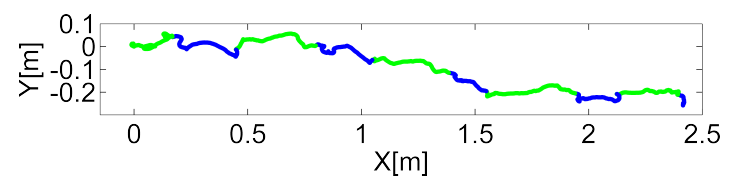

(a)

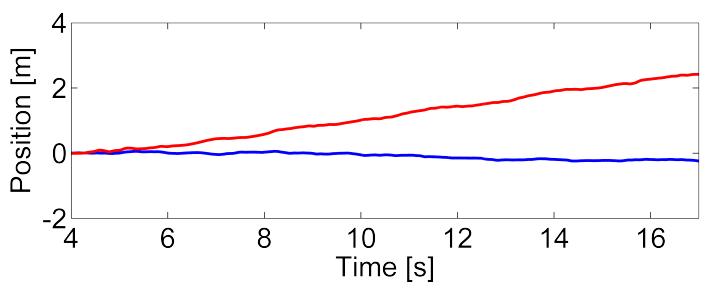

(b)

Figure 3: (a) Tracking data of walking along the X-direction with the current base foot color-coded. (b) Tracking position over time.

As shown in Figure 3, the system delivers reliable results in a preliminary system evaluation. The system is capable of correctly tracking a moving user. While the self-carried magnetic position tracker itself is drift-free, the presented tracking approach still accumulates errors over time as for all relative tracking approaches, i.e. the accuracy of the absolute position estimate degrades. However, this error only occurs at the base switch and is bounded by the measurement error of the position tracker. Thus, it does not increase quadratically as IMU tracking systems do. Hence, the error is not critical for virtual reality or human computer interaction applications as high precision and low latency is most important but not accuracy. If a higher accuracy is required, or for compensating the accumulated error, some fixed reference points might be installed. However, the effort for doing so is significantly smaller compared to existing absolute tracking systems

Future work will focus on determining the absolute tracking error as a function of time. For doing so, we will compare the tracking results with those stemming from the absolute measuring system Intersense IS1200.

\section{REFERENCES}

[1] E. Foxlin. Pedestrian tracking with shoe-mounted inertial sensors. Computer Graphics and Applications, 25(6):38-46, Nov.-Dec. 2005.

[2] A. Hamaguchi, M. Kanbara, and N. Yokoya. User localization using wearable electromagnetic tracker and orientation sensor. In Wearable Computers, 2006 10th IEEE International Symposium on, pages 55-58, Oct 2006.

[3] E. Hodgson, E. Bachmann, D. Vincent, M. Zmuda, D. Waller, and J. Calusdian. WeaVR: a self-contained and wearable immersive virtual environment simulation system. Behavior Research Methods, pages $1-12,2014$.

[4] K. Leuenberger and R. Gassert. Low-power sensor module for longterm activity monitoring. In Annual International Conference of Engineering in Medicine and Biology Society, pages 2237-2241. IEEE, 2011.

[5] R. A. Ruddle and S. Lessels. The benefits of using a walking interface to navigate virtual environments. ACM Transactions on Computer-Human Interaction, 16(1):1-18, 2009.

[6] K. Yamanaka, M. Kanbara, and N. Yokoya. Localization of walking or running user with wearable $3 \mathrm{~d}$ position sensor. In Artificial Reality and Telexistence, 17th International Conference on, pages 39-45, Nov 2007.

[7] M. Zank, T. Nescher, and A. Kunz. Robust prediction of auditory step feedback for forward walking. In Proceedings of the 19th ACM Symposium on Virtual Reality Software and Technology, pages 119-122. ACM, Oct. 2013. 